\title{
Seeing carbon emissions
}

Growing, harvesting and transporting $10 \mathrm{~kg}$ of potatoes in the UK has an average carbon footprint of 1.8 kilograms of carbon dioxide. A US couple getting divorced adds around 2.8 metric tonnes to their annual emissions. The world's seven billion inhabitants are pumping out somewhere in the region of 28 gigatonnes of carbon dioxide per year.

Figures for carbon costs and savings are used increasingly across a range of sectors, but for most people the numbers lack meaning. What does a gigatonne of carbon dioxide even look like?

Well, how about seeing the emissions next to the city where they are produced, as in the bottom image. It shows London's daily emissions of carbon dioxide 139 tonnes - scaled at atmospheric pressure filling a sphere of 521 metres across. Or more than two BT Towers high.

It's the work of British physicist turned artist Adam Nieman, who is trying to communicate carbon emission quantities in ways that non-specialists can get to grips with.

"If you are a scientist or a civil servant used to working with emissions, the questions you have can be answered most clearly by graphs and tables," says Nieman. "But plonking a graph in front of most people is not necessarily the best way to communicate. Our pictures are a way to talk about emissions quantifiably but not numerically, which engages people."

A key technique he uses is to place the volumes of carbon dioxide in familiar contexts to provide scale. "If you know what it is like to stand next to a landmark such as Big Ben, this can help you make sense of these quantities in a way that numbers and graphs can't unless you are used to working with them," says Nieman, who got a physics degree at Edinburgh University, and later went on to do a PhD in the visual culture of science.

Nieman started out creating art-based tools to help those without backgrounds in physics and mathematics to appreciate the scales involved in the universe. His Space Signpost project, for example, is a sign that re-orientates itself towards objects in space when passers-by select those objects from a touch screen. The first one was placed in Millennium Square, Bristol,

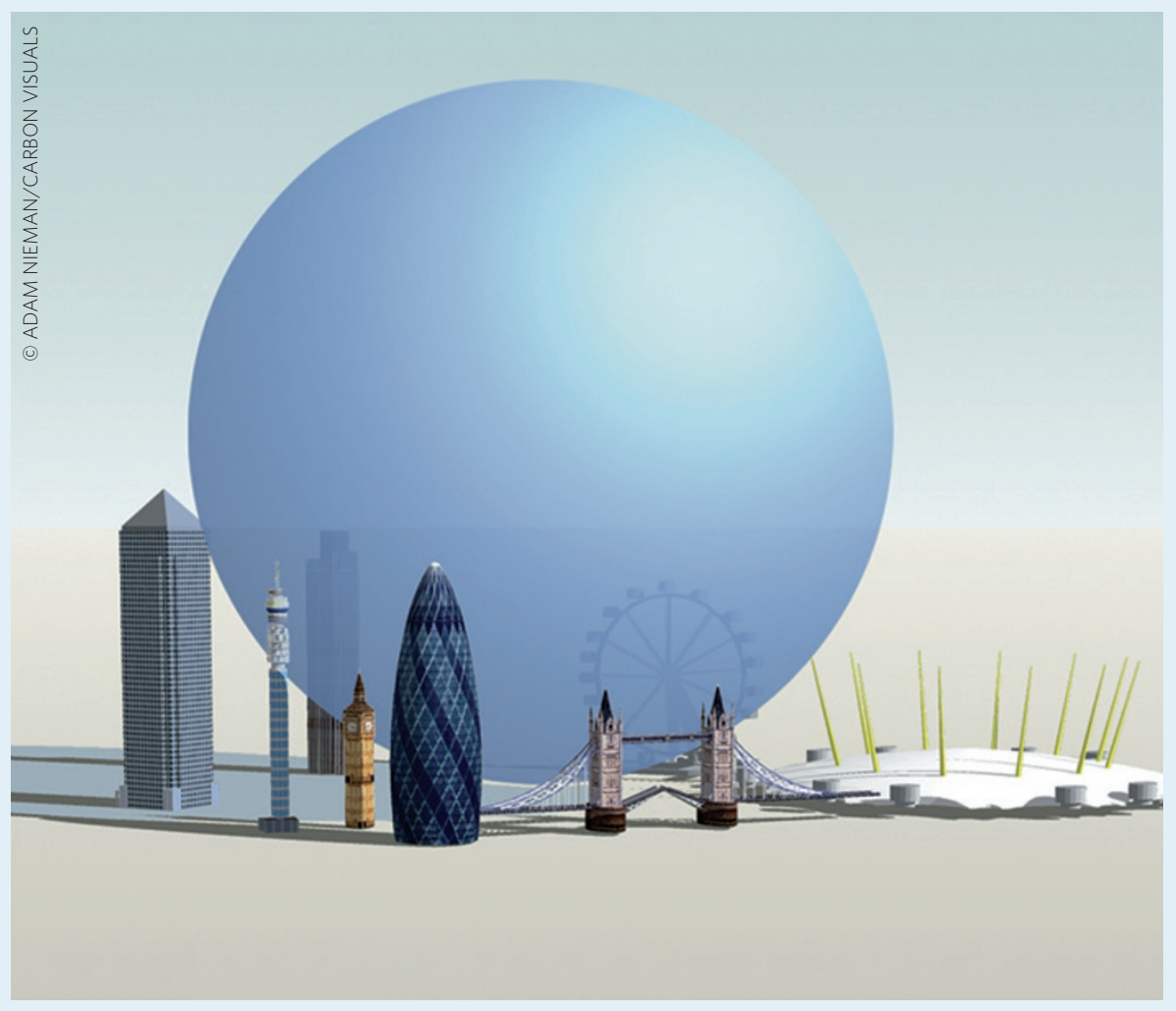

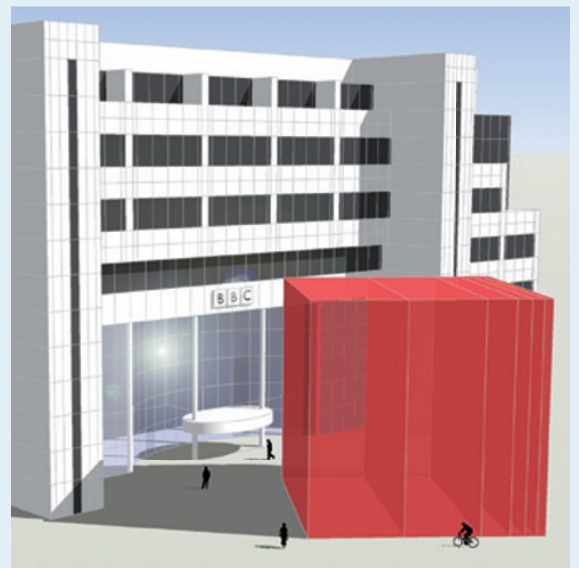

in 2006, and since then another has been erected at the Copernicus Science Centre, in Warsaw.

In recent years, Nieman has focused on carbon dioxide. In 2010, he helped set up Carbon Visuals - a five-person company that designs infographics, videos and online tools for organizations seeking to communicate carbon dioxide emissions, targets and savings.

In 2011, the group was commissioned by the BBC to create Albert - a carbon calculator designed to help those making television programmes work out and visualize the associated carbon costs. They calculated that making an hour of television generates an average of 8.2 tonnes of carbon dioxide. This is approximately equal to the annual emissions of two average houses in the UK and enough to fill a cube over 16 metres tall at sea-level pressure, at a temperature of $15^{\circ} \mathrm{C}-$ roughly half the height of the iconic $\mathrm{BBC}$ building at White City, as shown in the top image.

Carbon Visuals also created a short film as part of the BBC project (http:// go.nature.com/x4GKdk).

Nieman is now focusing on realtime visualizations. His latest project illustrates the carbon dioxide savings from solar panels at a farm in Devon, shown against the National Grid flow, which generates 5.8 tonnes of carbon dioxide every second. 


\section{Correction}

In the print version of the Snapshot 'Seeing carbon emissions' (N. Fleming, Nature Clim. Change 2,$306 ; 2012$ ), the credit for the top image should have read: (C) BBC. This is correct in the HTML and PDF versions. 